\title{
Hierarchical Model for Conditioning Information Signals at the MANET Physical Level with Ultraviolet Channel
}

\author{
G.S. Vasilyev ${ }^{1}$, O.R. Kuzichkin ${ }^{1}$, D.I. Surzhik ${ }^{2}$, I.S. Konstantinov ${ }^{1,3}$, S.A.Lazarev ${ }^{1}$ \\ ${ }^{1}$ Belgorod State University, Belgorod, 308015, Russia \\ ${ }^{2}$ Vladimir State University, Vladimir, 600000, Russia \\ ${ }^{3}$ Russian Timiryazev State Agrarian University, Moscow, 127550, Russia
}

\begin{abstract}
The use of mobile ad hoc networks (MANET) with a nonline-of-sight (NLOS) communication channel in the UV-C spectral range from 200 to $280 \mathrm{~nm}$ is advisable in a number of conditions when traditional radio communication is ineffective (strong interference due the electromagnetic background interference, challenging terrain etc.). The detailed development of a data transport physical layer model is required to create reliable and efficient MANETs with a UV channel; the model is determined by the operating conditions of the networks. A hierarchical model for the conditioning of information signals has been developed, and also an optical transmitter and an optical receiver in order to provide their analytical description and further analysis; the model allows one to present various options for communication channels. Analytical expressions are obtained for the transfer function of the generalized hierarchical model for an arbitrary number of open levels of the hierarchy. The energy characteristics of the UV communication system are calculated at various noise levels depending on the geometric parameters of the UV channel: communication range and azimuthal deviation of the transmitter and receiver.
\end{abstract}

Keywords: MANET, Physical layer, NLOS UV, Hierarchical model.

\section{INTRODUCTION}

The effectiveness of mobile ad hoc networks (MANET) is largely determined by the quality of solutions used in the design of the physical layer of such networks. The use of radio communication at the MANET physical level in the presence of obstacles between the transmitter and receiver, high electromagnetic background interference level, the creation of deliberate interference by means of electronic warfare, etc. is problematic. The use of optical communication systems is advisable when the communication is exposed to strong electromagnetic interference. However, infrared communication and data and acquisition (IrDA) $[1,2]$ and visible light communication (VLC) [3-5] only function in line of sight. The possibility of infrared communication due to reflection from the walls is real only in closed indoor conditions at extremely small distances of not more than $20 \mathrm{~m}$ [2]. Communication systems of the solar-blind ultraviolet UVC range from 200 to $280 \mathrm{~nm}$ provide the ability to communicate in the non-line-of-sight conditions (NLOS) due to strong scattering of UV radiation in the atmosphere, the communication range is up to $2-5 \mathrm{~km}[6,7]$. Thus, only this communication method is effective in the above difficult conditions.

To create a reliable MANET data transport architecture with a UV channel, a detailed model representation of the physical layer is required. Models of an optical transmitter, communication channel, and optical receiver are considered at this level. A large number of well-known models consider in detail NLOS options for UV channels with different geometries (different elevation angles and the pattern angle of the transmitter and the receiver), different weather conditions, medium turbulence, radiation polarization, etc. [8-13]. The functioning of UV communication means under the influence of various external and internal destabilizing factors (not only external optical noise caused by radiation from the Sun and other light sources, but also internal destabilizing factors affecting the optical transmitter and receiver units) has not been sufficiently studied. It is also problematic to carry out modelling taking into account the transfer functions of communication equipment, which cause distortion of information signals due to various inertia and nonlinearities of the constituent units.

The aim of the work is to develop a hierarchical model for the information signals generation for the digital and analytical modelling of MANET networks with a UV channel at the physical level.

\subsection{Physical implementation of a UV-C communication system for its use in MANET networks}

The general diagram of a single-channel UV communication system is shown in Fig. 1. 


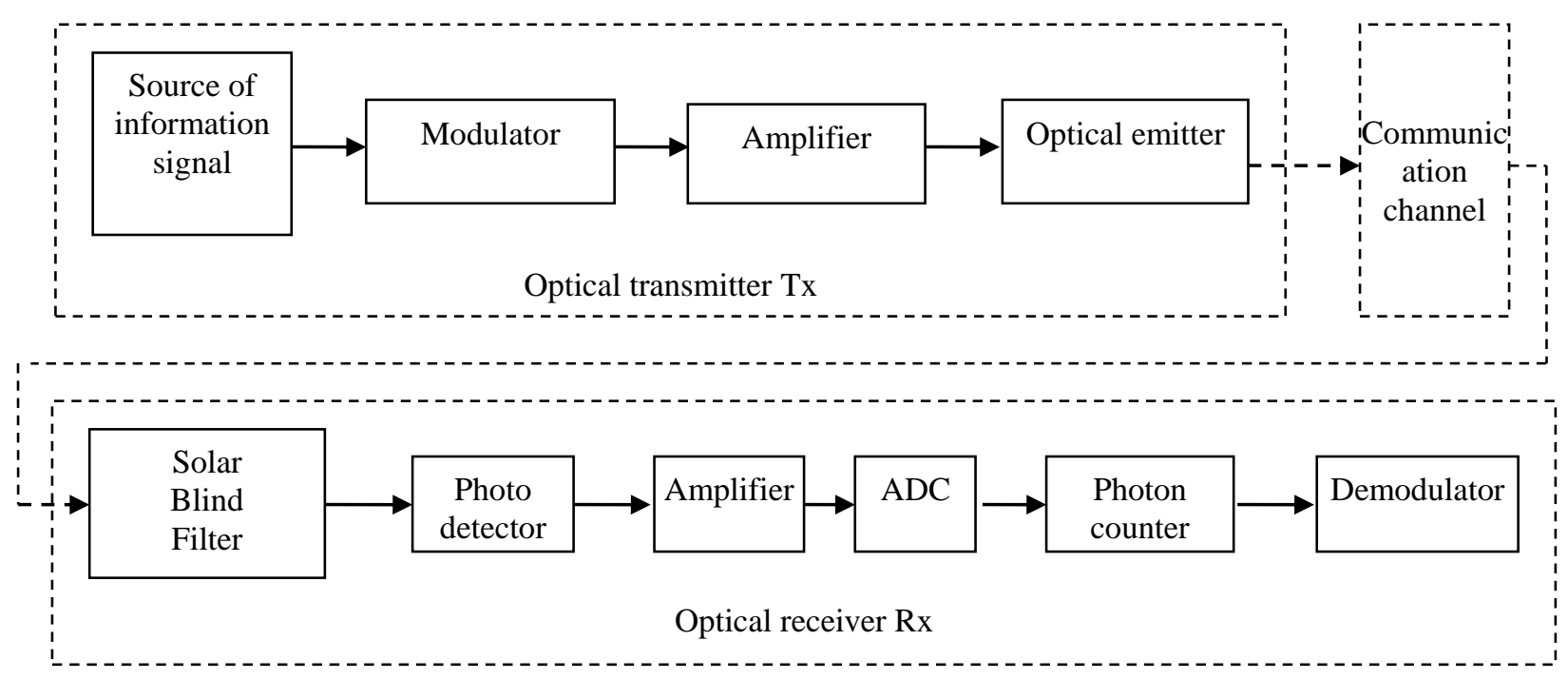

Fig. 1: General diagram of a single-channel UV communication system

UV lasers, single LEDs or LED arrays are used as optical emitters in UV communication systems. It is advisable to use a photomultiplier tube (PMT) as a optical receiver, because it is more sensitive than photodiodes. Considering the fragility of conventional PMTs, it is advisable to consider the choice of ruggedized PMTs for use in on-board mobile transport nodes $[14,15]$. A solar-blind absorption type filter with suppression of the visible range in more than 12 orders of magnitude is required at the output of the photomultiplier to effectively suppress solar radiation [16]. A transimpedance amplifier is used to amplify the optical receiver current [17]. The automatic gain control system with a large adjustment depth (up to $100 \mathrm{~dB}$ or more) as part of the receiver amplifier is necessary for receiving a signal with different levels, because attenuation in the NLOS UV channel varies from $60 \mathrm{~dB}$ to $160 \mathrm{~dB}$ or more, depending on the communication range of 1 $\mathrm{m} \ldots 4 \mathrm{~km}$ and other conditions [7]. The NLOS UV communication system must register single photons, so the analogue PMT mode is not applicable, and we need to use an analogue-to-digital converter (ADC) and a photon counter.

\subsection{The hierarchical model for the MANET information signal conditioning with ultraviolet channel}

The hierarchical model for the generation of information signals should allow the presentation of various options for communication channels and channel-forming equipment, to provide their analytical description and further analysis. The generalized model should provide the representation of UV communication channels between mobile nodes, taking into account the effects of attenuation, symbol-to-symbol interference, and multipath propagation of signals. In addition, the model should allow the study of network equipment circuits with linear and nonlinear signal conversion; circuits with feed-forward regulation (FFR), feedback regulation (FBR) and combined regulation; circuits with multi-channel signal conditioning and processing, as well as with cross-links between channels.
The generalized circuit of a transformer of signals (TS) (Fig. 1) contains similar SCs, a control unit (CU), control paths (CPs) and a weight distributor (WD). The generalized conditioner includes two control circuits: for disturbance compensation - feed-forward regulation (FFR), and for proportional or error-closing compensation - feedback regulation (FBR).

Each control path includes a detector and filter connected in series. As a detector, the conditioner model can contain various types of demodulators depending on the type of modulation used at the physical level: on-off keying (OOK), or pulse-position modulation (PPM); in addition, digital pulse interval modulation (DPIM and dual-head DPIM, DH-DPIM), which do not require external synchronization of the transmitter and receiver [18-23] could be used. The units are designated as $\mathrm{SC}^{\mathrm{x} 1}{ }_{\mathrm{x} 2}$, where $\mathrm{x} 1$ is the level number, $\mathrm{x} 2$ is the unit number. The symbols $U$ represent external main input and output signals, $\mathrm{u}$ symbols are for auxiliary signals. The upper and lower indices of the signals are designated as $\mathrm{U}^{\mathrm{y} 1}{ }_{\mathrm{y} 2 \mathrm{y} 3}$, where $\mathrm{y} 1$ is the level number, $\mathrm{y} 2$ is the number of the unit to which the signal belongs; y 3 is the signal identifier. The identifier y3 can take the following alphabetic and numerical designations: 1 - input signal of the unit, 2 - output, y - control signal CU, f - CPs of FFR circuit, b - CPs of FBR circuit.

The TS scheme can be expanded like a television raster by "lines" (expansion by levels) and by "frame". Fig. 1a and $1 \mathrm{~b}$ show the second and $\alpha(\alpha \geq 1)$ levels of TS expansion, respectively. Each expansion of the next level is carried out by expanding the contracted TS contained at the previous level. The level $\alpha=0$ corresponds to an unexpanded and contracted TS.

Denote the transfer functions of the units: TS $\rightarrow \Pi, \mathrm{CU} \rightarrow K$, $\mathrm{WD} \rightarrow n, \mathrm{CP} \rightarrow W$. Function indices are fully consistent with the indices of their units. In accordance with Fig. 1, the transfer functions of the TS look like: level $0 Q^{0}=\Pi^{0}{ }_{1}-$ contracted TS; level $1 Q^{1}=\Pi^{1}{ }_{1} K^{1}{ }_{1} \Pi^{1}{ }_{2}$; level $2 Q^{2}=\Pi^{2}{ }_{1} K^{2}{ }_{1} \Pi^{2}{ }_{2}$ $K^{1}{ }_{1} \Pi_{3}^{2} K_{2}{ }_{2} \Pi^{2}{ }_{4}$, etc. For the level $\alpha$ 
$Q^{\alpha}=\Pi_{\beta}^{\alpha-1}=\Pi_{2 \beta-1}^{\alpha} \mathrm{K}_{\beta}^{\alpha} \Pi_{2 \beta}^{\alpha}$
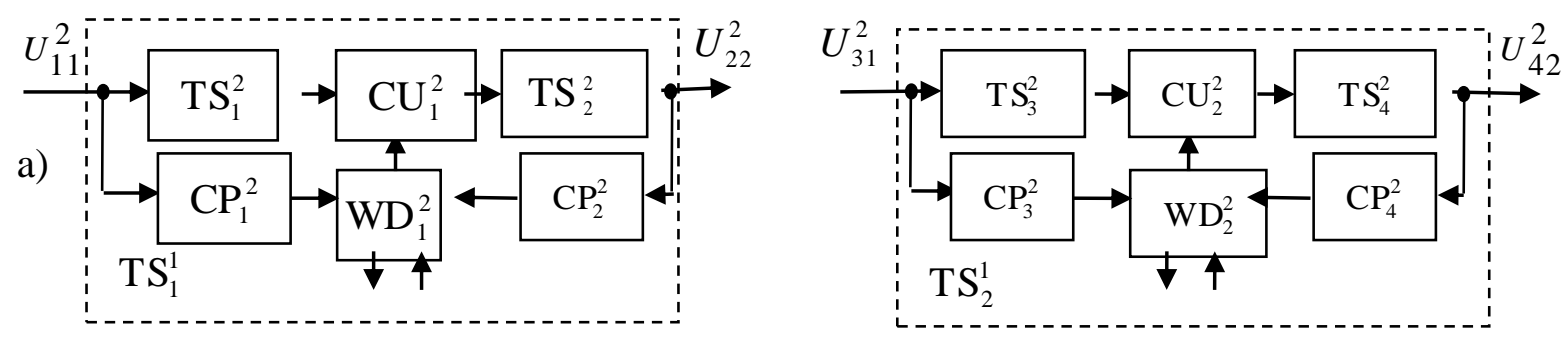

b)

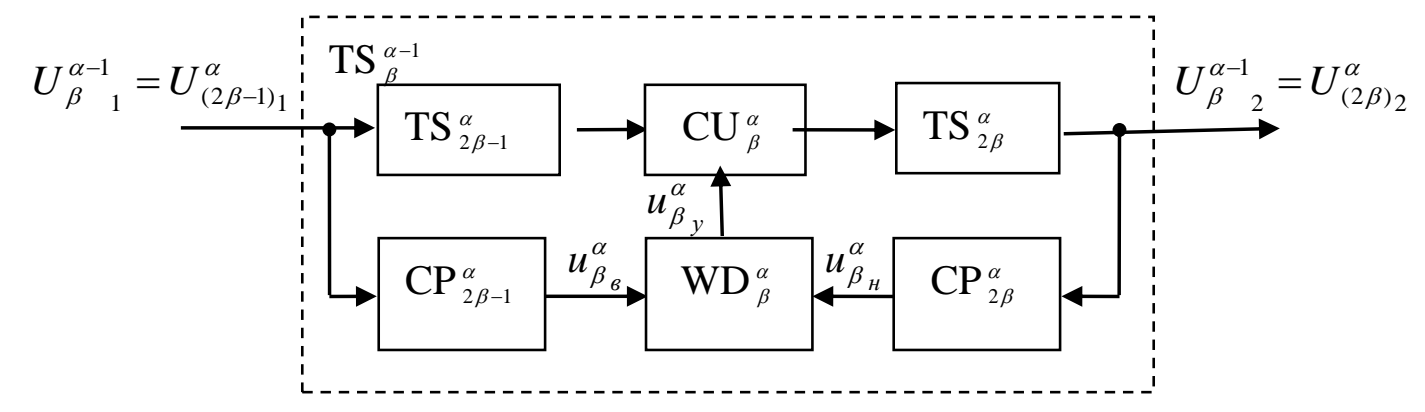

Fig. 2: Hierarchical model for generating information signals

Taking into account the transfer functions. WD and $\mathrm{CP}$ for the current number of the expanded layer

$\Pi_{\beta}^{\alpha-1}=U_{\beta}^{\alpha-1}{ }_{2} / U_{\beta}^{\alpha-1}{ }_{1}=\Pi_{2 \beta}^{\alpha}\left(\Pi_{2 \beta-1}^{\alpha}-\mathrm{f}_{\beta_{3}}^{\alpha} K_{\beta_{y}}^{\alpha}\right) /\left(1+\Pi_{2 \beta^{3}}^{\alpha} \hat{\beta}_{H}^{\alpha} K_{\beta_{y}}^{\alpha}\right),(2)$

Where $\mathrm{f}_{\beta_{6}}^{\alpha}=n_{\beta_{b}}^{\alpha} W_{2 \beta-1}^{\alpha}, \mathrm{f}_{\beta H}^{\alpha}=n^{\alpha}{ }_{\beta H} W^{\alpha}{ }_{2 \beta}$, - transfer functions of the FFR and FBR circuits, respectively, $\mathrm{K}^{\alpha}{ }_{\beta y}$ is the transfer function of the control unit (for the signal $u^{\alpha}{ }$ ).

Equating the right-hand sides of (1) and (2), we obtain the transfer function for the $\mathrm{CU}$

$$
\mathrm{K}_{\beta}^{\alpha}=\left(\Pi_{2 \beta-1}^{\alpha}-\mathrm{f}_{\beta_{B}}^{\alpha} K_{\beta}^{\alpha}\right) /\left(\Pi_{2 \beta-1}^{\alpha}\left(1+\Pi_{2 \beta^{\prime}}^{\alpha} \mathrm{f}_{\beta_{H}}^{\alpha} K_{\beta_{y}}^{\alpha}\right)\right)
$$

In the absence of feed-forward and feedback links, the expression (3) is simplified: $\Pi^{\alpha-1}{ }_{\beta}=\Pi^{\alpha}{ }_{2 \beta} \Pi^{\alpha}{ }_{2 \beta-1}$.

The proposed approach makes it possible to reduce the models of a specific channel or data transmission device in the MANET network to setting the transfer characteristics in the corresponding element of the "frame" of the generalized hierarchical model.

1.3. Modelling the energy characteristics of the NLOS UV communication system based on a hierarchical signal conditioning model

The energy characteristics of the on-of keying modulation with non-return to zero (NRZ-OOK) are given by [24]:

$$
B E R_{N R Z-O O K}(S N R)=\frac{1}{2} \operatorname{erfc}\left(\frac{1}{2 \sqrt{2}} \sqrt{S N R}\right) .
$$

The signal-to-noise ratio (SNR) of the UV channel is defined as the ratio of the number of detected signal photons $\mathrm{Nd}$ to the number of noise photons $N_{n}$ :

$$
S N R=N_{d} / N_{n}
$$

Where $N_{d}=\eta_{f} \eta_{r} N_{r}=\eta_{f} \eta_{r} N_{r} /$ Loss $=\eta_{f} \eta_{r} P_{T} \lambda /($ hcR $\cdot$ Loss $)$.

Here, $P_{T}$ is the transmitter radiation power, $\eta_{f}$ is the transmission coefficient of the sun-blind filter, $\eta_{r}$ is the quantum efficiency of the detector (receiver), $\eta_{r}$ is the number of received photons, $\lambda$ is the radiation wavelength, Loss is the channel loss, $R$ is the bitrate, $h=6.626 \cdot 10-34 \mathrm{~J} \cdot s$ is Planck's constant, $c=3 \cdot 10^{8} \mathrm{~m} / \mathrm{s}$ is the speed of light in vacuum. The measurement results showed that in the worst case, when the optical receiver is directed towards the sun in clear weather, the detection frequency of noise photons is about $15000 \mathrm{~Hz}$ (when using a high-quality solar-blind filter and a photomultiplier tube with an aperture of $1.92 \mathrm{~cm} 2$ ) [25]. Typical frequencies for detecting noise photons at low, medium and high noise levels are around 1000; 5000; 15000 $\mathrm{Hz}$, respectively [25].

When moving the MANET network nodes or changing the characteristics of the UV channel, an adaptive change in the transmitter power is necessary depending on specific conditions. According to (2), the radiation power sufficient to achieve a given SNR value is determined by the expression:

$$
P_{T}\left(r, \theta_{1}, \theta_{2}, \psi_{1}, \psi_{2}\right)=\frac{S N R \cdot N_{n} \cdot h c R \cdot \operatorname{Loss}\left(r, \theta_{1}, \theta_{2}, \psi_{1}, \psi_{2}\right)}{\eta_{f} \eta_{r} \lambda} .
$$

The energy characteristics of the UV communication system calculated by expression (3) are shown in Fig. 2. Modelling 
the values of losses in the UV channel is based on the Monte Carlo method. The following UV channel parameters were adopted: communication range $\mathrm{r}=100 \mathrm{~m}$, elevation angles of the transmitter and receiver $\theta 1=30^{\circ}$ and $\theta 2=30^{\circ}$, widths of radiation patterns of the transmitter and receiver $\varphi_{1}=10^{\circ}$ and $\varphi_{2}=30^{\circ}$, radiation wavelength $\lambda=260 \mathrm{~nm}$, scattering and absorption coefficients for clear weather, receiver aperture area $\mathrm{Ar}=1.92 \mathrm{~cm}^{2}$ ). Also the values of $\mathrm{SNR}=10 \mathrm{~dB}, \mathrm{R}=100$ $\mathrm{kbit} / \mathrm{s}$ are accepted.
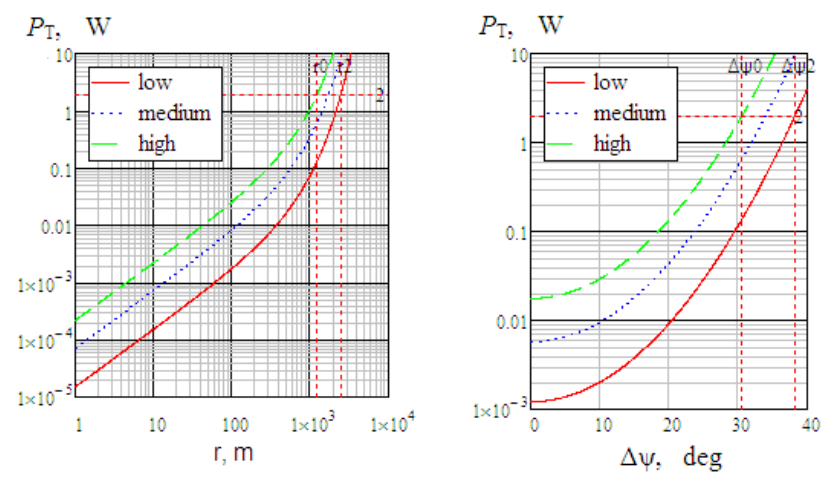

Fig. 3: Energy characteristics of the UV communication system at different noise levels: a) depending on the range $r$; b) depending on the azimuthal deviation $\Delta \psi=\left|\psi_{1}\right|+\left|\psi_{2}\right|$

The maximum power of UV-C LEDs is $130 \mathrm{~mW}$ (SMD 6060 UVC LED High-Power) versus $1 \mathrm{~W}$ for UV-C lasers. To increase the radiation power, it is advisable to use LED arrays with an emitted power of up to $2 \mathrm{~W}$ (FLS 6060 UVC SMD LED 5x5 Array). According to fig. $2 \mathrm{a}$, at $\mathrm{P}_{\mathrm{Tmax}}=2 \mathrm{~W}$ and the selected characteristics of the channel and transmission mode, the limiting range is $1250 \mathrm{~m}, 1720 \mathrm{~m}$, and $2490 \mathrm{~m}$ at high, medium, and low noise levels, respectively. According to fig. $2 \mathrm{~b}$, at $\mathrm{P}_{\mathrm{T} \max }=2 \mathrm{~W}$, communication at a distance of $\mathrm{r}=100 \mathrm{~m}$ is ensured with an azimuthal deviation of not more than $30^{\circ}$, $34^{\circ}$, and $38^{\circ}$ at high, medium, and low noise levels, respectively. The studies carried out make it possible to determine the energy characteristics of the UV communication system under various operating conditions (when changing the state of the channel, transmission mode, rotations or movements of communication nodes).

\section{CONCLUSION}

A hierarchical model for the conditioning of information signals MANET with an NLOS UV channel has been developed; it allows one to present various options for communication channels. An optical transmitter and an optical receiver have been also developed to provide their analytical description and further analysis. Analytical expressions are obtained for the transfer function of the generalized hierarchical model for an arbitrary number of open levels of the hierarchy. On the basis of the proposed hierarchical signal conditioning model, the energy characteristics of the UV communication system are calculated at various noise levels depending on the geometric parameters of the UV channel: communication range and azimuthal deviation of the transmitter and the receiver.

\section{ACKNOWLEDGMENTS}

This work was supported by the Ministry of Science and Higher Education of the Russian Federation under Grant RFMEFI57518X0175 "Development of digital communication modules of mobile devices operating on the basis of UV data channels for establishing special purpose wireless ad-hoc networks".

\section{REFERENCES}

[1] Al-Nassar, Suha \& Hatem, Haraa \& Shehab, Jinan. (2018). Design and Implementation of Infrared (IR) Communication System. 10.26367/DJES/VOL.11/NO.3/5.

29-33.

[2] Arvin, Farshad \& Samsudin, Khairulmizam \& Ramli, Abdul. (2009). A Short-Range Infrared Communication for Swarm Mobile Robots. Signal Processing Systems, International Conference on. 454-458. 10.1109/ICSPS.2009.88.

[3] Ndjiongue, Alain Richard \& Ferreira, Hendrik \& Ngatched, Telex. (2015). Visible Light Communications (VLC) Technology. Wiley Encyclopedia of Electrical and Electronics Engineering. 1-15. 10.1002/047134608X.W8267.

[4] Giustiniano, Domenico; Tippenhauer, Nils Ole; Mangold, Stefan (2012). "Low-complexity Visible Light Networking with LED-to-LED communication". 2012 IFIP Wireless Days. pp. 1-8. doi:10.1109/WD.2012.6402861. ISBN 978-1-46734404-3.

[5] IEEE 802.15 WPAN Task Group 7 (TG7) Visible Light Communication

[6] Xu Z. and Sadler B. Ultraviolet communications: potential and state-of-the-art IEEE Commun. Mag.4667-73, 2009.

[7] Chen G, Liao L, Li Z, et al. Experimental and simulated evaluation of long distance NLOS UV communication $[\mathrm{C}] / /$ Communication Systems, Networks \& Digital Signal Processing (CSND-SP), 2014 9th International Symposium on. IEEE, 2014: 904-909.

[8] Ding H, Chen G, Majumdar A K, et al. Modeling of non-line-of-sight ultraviolet scattering chan-nels for communication[J]. Selected Areas in Communications, IEEE Journal on, 2009, 27(9): 1535-1544

[9] Han D, Liu Y, Zhang K, et al. Theoretical and experimental research on diversity reception technology in NLOS UV communication system[J]. Optics express, 2012, 20(14): 15833-15842.

[10] Ding H, Chen G, Majumdar A K, et al. Turbulence modeling for non-line-of-sight ultravi-olet scattering channels[C]//SPIE. 2011, 8038: 80380J.

[11] Konstantinov I.S., Vasilyev G.S., Kuzichkin O.R., Surzhik D.I., Lazarev S.A.. Numerical and Analytical 
Modeling of Wireless UV Communication Channels for the Organization of Wireless Ad-Hoc Network // IJCSNS - International Journal of Computer Science and Network Security - 2018. - Vol. 18, No. 8, pp. 98$104 . \quad$ Open access: http://paper.ijcsns.org/07_book/201808/20180815.pdf.

[12] I.S. Konstantinov, G.S. Vasilyev, O.R. Kuzichkin, I.A. Kurilov, S.A. Lazarev. Modeling and Analysis of the Characteristics of Ultraviolet Channels under Different Conditions of Radiation Propagation for the Organization of Wireless AD-HOC Network // JARDCS - Journal of Advanced Research in Dynamical and Control Systems - 2018. - 07-Special Issue, pp. 1853-1859. http://jardcs.org/abstract.php?archiveid=5147.

[13] Igor S. Konstantinov, G. S. Vasyliev, Oleg R. Kuzichkin, D.I. Surzhik, I. A. Kurilov, Sergey A. Lazarev. Development Of UV Communication Channels Characteristics Modeling Algorithm In A Mobile Ad-Hoc Network / Journal of Advanced Research in Dynamical and Control Systems (JARDCS) / ISSN: 1943-023X / Volume 11 | 08Special Issue, 2019. Pages: 1920-1928. http://www.jardcs.org/abstract.php?id=2543

[14] Hamamatsu Photomultiplier Tubes: basics and applications. - 4th edition, - P. 258.

[15] Bicron Corp.: Ruggedized High-Temperature Detector Technology.

[16] Ofil's Solar blind UV filters: http://www.sbuv.com/filters/sb268.html.

[17] Eduard Säckinger. Analysis and Design of Transimpedance Amplifiers for Optical Receivers, 2007, 592 p, ISBN: 978-1-119-26441-5.

[18] Xu C. and Zhang H., "Packet error rate analysis of IM/DD systems for ultraviolet scattering communications", IEEE Military Communications Conference (2015), pp. 1188-1193.

[19] Ghassemlooy Z., Kaluarachchi E. D., Reyher R. U., and Simmonds A. J., "A new modulation technique based on digital pulse interval modulation (DPIM) for optical-fiber communication," Microw. Opt. Technol. Lett., vol. 10, no. 1, pp. 1-4, Sep. 1995.

[20] Ghassemlooy Z., and Hayes A. R., "Digital pulse interval modulation for optical communications," IEEE Commun. Mag., vol. 36, no. 12, pp. 95-99, Dec. 1998.

[21] Hu Z., Tang J., "Performance of digital pulse interval modulation of atmospheric optical wireless communication system," Proc. SPIE, vol. 5625, pp. 202-208, Feb. 2005.

[22] Aldibbiat N. M., Ghassemlooy Z., and McLaughlin R., "Error performance of dual header pulse interval modulation (DH-PIM) in optical wireless communications," IEE Proc. Optoelectron., vol. 148, no. 2, pp. 91-96, Apr. 2001.
[23] Ma J., Jiang Y., Yu S., Tan L., and Du W., "Packet error rate analysis of OOK, DPIM and PPM modulation schemes for ground-to-satellite optical communications," Opt. Commun., vol. 283, pp. 237 242, Jan. 2010.

[24] Elganimi T.Y. Performance Comparison between OOK, PPM and PAM Modulation Schemes for Free Space Optical (FSO) Communication Systems: Analytical Study. International Journal of Computer Applications 79(11):22-27, October 2013.

[25] Chen G., F. Abou-Galala, Z. Xu, and B. M. Sadler, "Experimental evaluation of LED-based solar blind NLOS communication links," Optics Express, vol. 16, no. 19, pp. 15059-15068, Sep. 2008. 